\title{
Role of Positron Emission Tomography/Computed Tomography in The Initial Staging And Evaluation Of The Therapeutic Response in Patients with Non-Hodgkin Lymphoma
}

\author{
Hesham E. ELsheikh, Mohamed A. Attia, Adel M. Kotb
}

\begin{abstract}
Department of RadioDiagnosis and Medical Imaging, Faculty of Medicine Benha University, Egypt .

Correspondence to: Adel M. Kotb. Department of Radio- Diagnosis and Medical Imaging, Faculty of Medicine Benha University, Egypt .
\end{abstract}

Email:

adelkotbbenha@yahoo.com

Received: 3 July 2021

Accepted: 16 August 2021

\begin{abstract}
Background: Malignant lymphoma is the most common hematological malignancy accounts for approximately $8 \%$ of all adult malignancies. The aim of this work is to study the role of PET/CT in the diagnosis and follow up of Non-hodgkin lymphoma. Methods: The study was conducted on eighty patients where the diagnosis of non-hodgkin lymphoma have been pathologically confirmed, 50 males $(62.5 \%)$ and 30 females $(37.5 \%)$, their ages are ranged between 14 to 81 years old. All patients were examined using Siemens Bio-graph true point PET/CT scanner. These dedicated systems integrate a PET scanner with a multi-slice helical CT scanners permit the acquisition of co-registered CT and PET images in one session. Results: among the 40 patients referred for follow up assessment, their SUVmax of the initial PET/CT examination ranged from $3-49.0$ with a mean value of $17.05 \pm 11.86 \mathrm{SD}$, with a significant change $(\mathrm{p} \leq 0.046)$ in their follow up examination with SUVmax ranged from $0.0-28.0$ with a mean value of $11.73 \pm 9.25 \mathrm{SD}$, There were statically significant difference between (PET/CT) staging and CECT scan staging $(\mathrm{p}<0.001)$. Conclusion: PET/CT proved statistically to be more efficient in follow up studies after chemotherapy using the standard uptake value (SUV) being more related to the functional activity of the residual tumor cells rather than to the size of the tumor itself.
\end{abstract}

Keywords: PET; CT; non-Hodgkin; Lymphoma 


\section{Introduction}

Malignant lymphoma is the most common hematological malignancy accounts for approximately $8 \%$ of all adult malignancies. Lymphomas are broadly divided into Hodgkin lymphoma and non-Hodgkin's lymphoma. Non-Hodgkin lymphoma accounts for about $5 \%$ of all cases of cancer .Non-Hodgkin lymphomas have the vast majority of cases and have a greater predilection to disseminate to extra-nodal sites (1).

18-fluorodeoxyglucose(18F-FDG) is a glucose analog that provides information about glucose metabolism of normal and abnormal tissues; it is taken up and accumulated in the malignant cells relatively mora than nonmalignant cells, However, the PET lacks the morphological information and has low anatomical resolution, thus fusion of the PET images and CT images that was obtained at the same position provides the precise anatomical ,morphological and functional information about the lesions with high glucose metabolism (2).

PET/CT scanning allows us to visualize and measure the intensity of residual metabolic activity within the lesion as well as estimating its size. Non-Hodgkin's lymphoma is FDG avid, and PET/CT is a more accurate in the initial staging than the conventional diagnostic CT, especially to detect unexpected sites of extranodal lymphoma (3).

However, caution must be exercised in the interpretation of PET scans because of technical limitations, variability of FDG avidity among the different lymphoma histological subtypes, and in the large number of etiologies of false-negative and false positive results. Recent attempts to standardize PET in clinical trials and incorporation of this technology into uniformly adopted response criteria will hopefully lead to improved outcome for patients with lymphoma (4).

PET-CT provides the needed information about both the metabolic activity as well the anatomical location of the neoplasm combining the advantages of both conventional methods CT and PET (5).

The aim of this work is to study the role of PET/CT in the diagnosis and follow up of Non-hodgkin lymphoma.

\section{Patients and methods}

This cross-sectional observational study was conducted on eighty patients where the diagnosis of non-hodgkin lymphoma have 
been pathologically confirmed, 50 males $(62.5 \%)$ and 30 females (37.5\%), their ages are ranged between 14 to 81 years old.

The study was conducted on eighty patients where the diagnosis of Non- Hodgkin lymphoma have been pathologically confirmed, referred to private center for PET/CT assessment in the period from 1/2019 till 5/2021, in Radiology department of Benha university hospitals

The study was approved by the Ethical committee of Benha faculty of Medicine, an informed consent was obtained from all patients in this research.

Patients were subjected to the following:

1. Full history taking.

2. Laboratory Testing.

3. Biopsy and histopathology (either FNAC or excisional biopsy).

\section{CECT scan.}

\section{PET/CT examination:}

Exams were done and data were obtained using Siemens Bio-graph true point PET/CT scanner. These dedicated systems integrate a PET scanner with a multi-slice helical CT scanners permit the acquisition of coregistered CT and PET images in one session.

\section{Statistical analysis}

Data were collected, coded, revised and entered to the Statistical Package for Social Science (IBM SPSS) version 20. The data were presented as number and percentages for the qualitative data, mean, standard deviations and ranges for the quantitative data with parametric distribution and median with inter quartile range (IQR) for the quantitative data with non parametric distribution.

Chi-square test was used in the comparison between two groups with qualitative data and Fisher exact test was used instead of the Chisquare test when the expected count in any cell found less than 5. Independent t-test was used in the comparison between two groups with quantitative data and parametric distribution and Mann-Whitney test was used in the comparison between two groups with quantitative data and non parametric distribution. The confidence interval was set to $95 \%$ and the margin of error accepted was set to $5 \%$. So, the p-value was considered significant as the following: $\mathrm{P}>0.05$ : Non significant (NS), P < 0.05: Significant (S), P < 0.01: Highly significant (HS) 


\section{Results}

The studied patients included 50 males $(62.5 \%)$ and 30 females (37.5\%), their ages ranged between 14 to 81 years with a mean age of $57 \pm 13$ years. Out of the 80 studied patients, 68 patients $(85 \%)$ were of $\mathrm{B}-$ cell type, 12 patients were $(15 \%)$ of $\mathrm{T}$ - cell type. In B-cell type there were $2.5 \%$ were burkitt's, $45 \%$ were DLBC, $25 \%$ were follicular and $7.5 \%$ were MALT, figure 1.

All patients presented with a range of clinical occurring separately or in conjunction. The commonest clinical presentations were painless swelling of lymph nodes in 44 patients $(55 \%)$ and fever in 30 patients $(37.5 \%)$.

In $\mathrm{H} \& \mathrm{~N}$ : all comparison were not significant. In Chest: There were no statically significant difference between PET/CT and CECT among GIT so there wasn't any statically significant difference in sub items. In MSK: There were statically significant difference between PET/CT and CECT among MSK because there was statically significant difference in BM, and subcutaneous. Finally in Abdomen: There were no statically significant difference between PET/CT and CECT among Abdomen so there wasn't any statically significant difference in sub items. table 1.

In our study, among the 40 patients referred for follow up assessment, their SUVmax of the initial PET/CT examination ranged from 3 - 49.0 with a mean value of $17.05 \pm 11.86$ $\mathrm{SD}$, with a significant change $(\mathrm{p} \leq 0.046)$ in their follow up examination with SUVmax ranged from $0.0-28.0$ with a mean value of $11.73 \pm 9.25 \mathrm{SD}$, table 2

Among the 80 cases referred for initial staging and according to the Ann Arbor classification, the cases were staged according to the size in CECT and FDG activity in PET/CT. PET detected 6 patients $(7.5 \%)$ in stage I, 16 patients (20\%) in stage II, 8 patients (10\%) in stage IIE, 12 patients (15\%) in stage III, 4 patients (5\%) in stage IIIE and 34 patients $(42.5 \%)$ in stage IV. The CT detected 8 patients (10\%) in stage I, 2 patients $(2.5 \%)$ in stage IE, 24 patients (30\%) in stage II, 6 patients $(7.5 \%)$ patients in stage IIIE and 20 patients $(2.5 \%)$ in stage IIII and IV, therefore PET/CT was statistically significant at $\mathrm{p} \leq 0.001$ in the initial staging. There were statically significant difference between (PET/CT) staging and CECT scan staging, table 3 
Among the 40 patients referred for follow up assessment after treatment according to the size in CECT and FDG activity in PET/CT ; CECT showed 18 patients $(22.5 \%)$ in regressive disease, 4 patients $(5 \%)$ in stable disease , 4 patient (5\%) in progressive disease and 14 patients $(17.5 \%)$ in remission, while PET/CT showed 20 patients (25\%) in regressive disease, 2 patients $(2.5 \%)$ in stable disease and 2 patient $(2.5 \%)$ in progressive disease 16 patients $(20 \%)$ in remission. table 4

CASE I: figure 2; A 47 years old male patient presented with painless right cervical swelling and pathologically proved DLBC NHL from right cervical nodal biopsy. PET/CT dated 3/9/2019 revealed: CT revealed: STAGE II Lymphoma with involvement of right deep cervical lymph node group as well as right axillary lymph node. PET/CT revealed; Stage I Lymphoma with active single lymph nodes group seen at the right deep cervical Lymph nodes while the right axillary lymph node with no tracer uptake.

CASE II: figure 3; A 73 years old male patient presented with anorexia and fever and diagnosed with of MALEFT gastric NHL after endoscopic excisional biopsy from the stomach. PET/CT 24/11/2019 revealed: CT revealed: Stage I MALEFT lymphoma with splenic focal involvement while stomach shows average wall thickening with no focal lesion could be noted. PET/CT revealed: Stage IE MALEFT lymphoma with active splenic focal lymphomatous infiltrate and extra nodal active focal gastric infiltrate. 
Benha medical journal, vol. 39, special issue (radiology), 2022

Table (1): Extra nodal lesions detected by PET/CT in comparison to detected by CECT

\begin{tabular}{|c|c|c|c|c|c|c|}
\hline & \multicolumn{2}{|c|}{ PET/CT } & \multicolumn{2}{|c|}{ CECT } & \multicolumn{2}{|c|}{ Chi square test/ Fisher test } \\
\hline & No & $\%$ & No & $\%$ & $\mathrm{X}^{2}$ & P value \\
\hline $\mathbf{H \& N}$ & 8 & $8.9 \%$ & 8 & $15.4 \%$ & 1.491 & . Tr人 \\
\hline Parotid & 2 & $2.50 \%$ & 2 & $2.50 \%$ & $\because \cdots$ & ${ }^{F}{ }_{p}=1 . \cdots$ \\
\hline Tonsils & 4 & $5.00 \%$ & 4 & $5.00 \%$ & $\because \cdots$ & $\mathrm{FE}_{\mathrm{p}=1}^{\mathrm{L}}=1$. \\
\hline Nasopharynx & 2 & $2.50 \%$ & 2 & $2.50 \%$ & $\because \cdots$ & ${ }_{\mathrm{E}}^{\mathrm{N}}=1, \ldots$ \\
\hline Chest & 20 & $22.2 \%$ & 16 & $30.8 \%$ & $1 . K V Y$ &.$r \circ 9$ \\
\hline Lung & 16 & $20 \%$ & 14 & $17.50 \%$ & $1.70 \leqslant$ & .191 \\
\hline Pleural effusion & 2 & $2.50 \%$ & 2 & $2.50 \%$ & $\because .07$ & ${ }^{F E} p=\cdot \lambda / r$ \\
\hline Breast & 2 & $2.50 \%$ & 0 & $0 \%$ & $1.79 \leq$ & $\mathrm{FE}_{\mathrm{p}}=.19 \pi$ \\
\hline MSK & 26 & $28.9 \%$ & 6 & $11.5 \%$ & $0.71 \mu$ & $\because \cdot 1 \mathrm{~V}$ \\
\hline $\mathrm{BM}$ & 14 & $17.50 \%$ & 0 & $0 \%$ & $0 . V \leq \varepsilon$ & ${ }^{F} E_{p}=\cdot . \cdot 17$ \\
\hline Muscles & 4 & $5.00 \%$ & 0 & $0 \%$ & 1.00 & $\mathrm{FE}_{\mathrm{p}}^{L}=\cdot r \cdot \Sigma$ \\
\hline Cortical bone & 2 & $2.50 \%$ & 2 & $2.50 \%$ & r.qr. & $\mathrm{FE}_{\mathrm{p}}^{\mathrm{T}}=\cdot . \wedge \mathrm{t}$ \\
\hline Subcutanous & 6 & 7.5 & 4 & $5 \%$ & $\varepsilon . M$ & $\mathrm{FE}_{\mathrm{p}}^{\mathrm{L}}=\cdot . \cdot \mathrm{rV}$ \\
\hline Abdomen & 36 & $40 \%$ & 22 & $42.3 \%$ & $\because V T$ & $\because \vee \wedge \vee$ \\
\hline Liver & 4 & $5.00 \%$ & 0 & $0 \%$ & Tצד & ${ }^{F E} \mathrm{p}=.1 .0$ \\
\hline Spleen & 22 & $27.5 \%$ & 16 & $20.00 \%$ & $\cdot \wedge 17$ & • \\
\hline Stomach & 4 & $5 \%$ & 2 & $2.50 \%$ & $\because 7$ & $\left.{ }^{F E} \mathrm{p}=\cdot . \wedge \cdot\right\urcorner$ \\
\hline Intestines & 4 & $5 \%$ & 2 & $2.50 \%$ & $\because 7$ & $\left.\mathrm{FE}_{\mathrm{p}}^{\mathrm{L}}=\cdot . \wedge \cdot\right\urcorner$ \\
\hline Adrenal & 2 & $2.50 \%$ & 2 & $2.50 \%$ & • & $\mathrm{FE}_{\mathrm{p}}^{\mathrm{L}}=\cdot .7 \cdot 7$ \\
\hline Total & 90 & $100 \%$ & 52 & $57.8 \%$ & 48.169 & 0.001 \\
\hline
\end{tabular}

$\chi^{2}$ : Chi square test FE: Fisher Exact p: p value for comparing between PET/CT and CECT

Table (2): $\quad$ Descriptive of the studied cases according to SUVmax value $(n=20)$

\begin{tabular}{|c|c|c|c|c|}
\hline & SUVmax Baseline & SUVmax Follow up & $\mathbf{Z}$ & $\mathbf{P}$ \\
\hline \multicolumn{5}{|l|}{ SUVmax } \\
\hline Min. - Max. & $3-49$ & $0.0-28.0$ & & \\
\hline Mean \pm SD & $17.05 \pm 11.86$ & $11.73 \pm 9.25$ & $1.998^{*}$ & $0.046^{*}$ \\
\hline Median & 14 & 9.50 & & \\
\hline
\end{tabular}

$\mathrm{Z}, \mathrm{p}: \mathrm{Z}$ and $\mathrm{p}$ values for Wilcoxon signed ranks test for comparing between baseline and follow up *: Statistically significant at $\mathrm{p} \leq 0.05$ 
Table (1): Comparison between (PET/CT) staging and CECT scan staging $(\mathrm{n}=80)$

\begin{tabular}{|c|c|c|c|c|c|c|c|c|c|c|c|c|c|}
\hline & & \multicolumn{12}{|c|}{ By PET } \\
\hline & & \multicolumn{2}{|c|}{ Stage I } & \multicolumn{2}{|c|}{ Stage II } & \multicolumn{2}{|c|}{ Stage IIE } & \multicolumn{2}{|c|}{ Stage III } & \multicolumn{2}{|c|}{ Stage IIIE } & \multicolumn{2}{|c|}{ Stage IV } \\
\hline & & No & $\%$ & No & $\%$ & No & $\%$ & No & $\%$ & No & $\%$ & No & $\%$ \\
\hline & Stage I & 2 & $33.3 \%$ & 2 & $12.5 \%$ & 2 & $25.0 \%$ & 0 & $0.0 \%$ & 0 & $0.0 \%$ & 2 & $5.9 \%$ \\
\hline & Stage IE & 0 & $0.0 \%$ & 0 & $0.0 \%$ & 2 & $25.0 \%$ & 0 & $0.0 \%$ & 0 & $0.0 \%$ & 0 & $0.0 \%$ \\
\hline & Stage II & 4 & $66.7 \%$ & 10 & $62.5 \%$ & 4 & $50.0 \%$ & 6 & $50.0 \%$ & 0 & $0.0 \%$ & 0 & $0.0 \%$ \\
\hline & Stage IIE & 0 & $0.0 \%$ & 0 & $0.0 \%$ & 0 & $0.0 \%$ & 0 & $0.0 \%$ & 2 & $50.0 \%$ & 4 & $11.8 \%$ \\
\hline & Stage III & 0 & $0.0 \%$ & 4 & $25.0 \%$ & 0 & $0.0 \%$ & 6 & $50.0 \%$ & 2 & $50.0 \%$ & 8 & $23.5 \%$ \\
\hline & Stage IV & 0 & $0.0 \%$ & 0 & $0.0 \%$ & 0 & $0.0 \%$ & 0 & $0.0 \%$ & 0 & $0.0 \%$ & 20 & $58.8 \%$ \\
\hline Chi square & 95.928 & & & & & & & & & & & & \\
\hline P value & 0.001 & & & & & & & & & & & & \\
\hline
\end{tabular}

Table (4): Distribution of the studied cases according to follow up treatment and disease response $(n=40)$

\begin{tabular}{|c|c|c|c|}
\hline & & No & $\%$ \\
\hline \multirow{4}{*}{ PET/CT follow up } & Progressive & 2 & $5 \%$ \\
\hline & Regressive & 20 & $50 \%$ \\
\hline & Remission & 16 & $40 \%$ \\
\hline & Stable & 2 & $5 \%$ \\
\hline \multirow{4}{*}{ CT follow up } & Progressive & 4 & $10 \%$ \\
\hline & Regressive & 18 & $45 \%$ \\
\hline & Remission & 14 & $35 \%$ \\
\hline & Stable & 4 & $10 \%$ \\
\hline
\end{tabular}




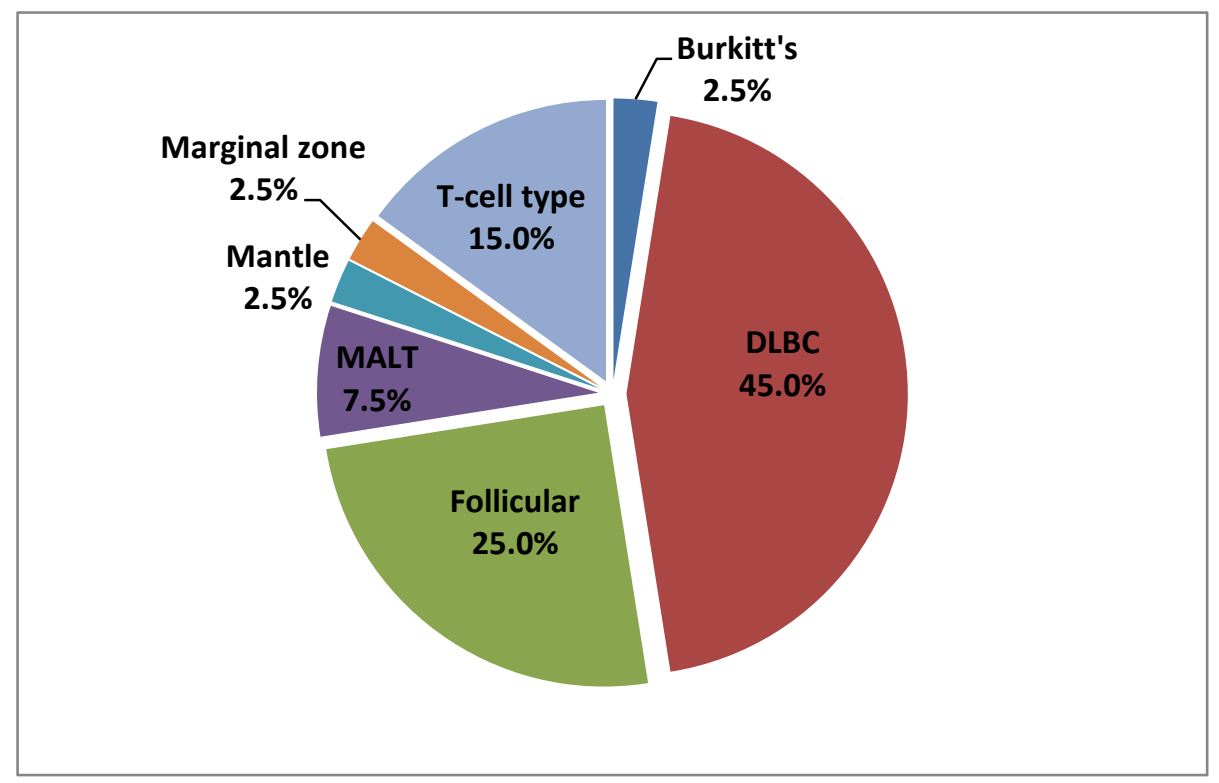

Figure (1): Distribution of Pathological type of NON-Hodgkin lymphoma

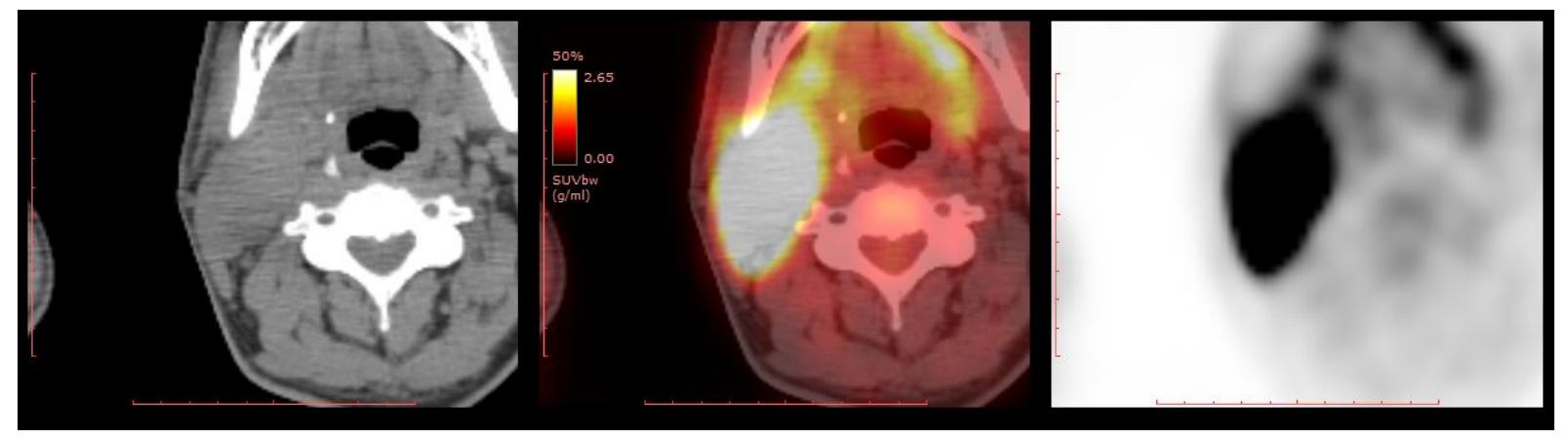

Fig. (2)(a): Multiple amalgamated right deep cervical LNs level II measuring up to (5x $3 \mathrm{~cm})$, eliciting dense tracer fixation with SUVmax up to 35.

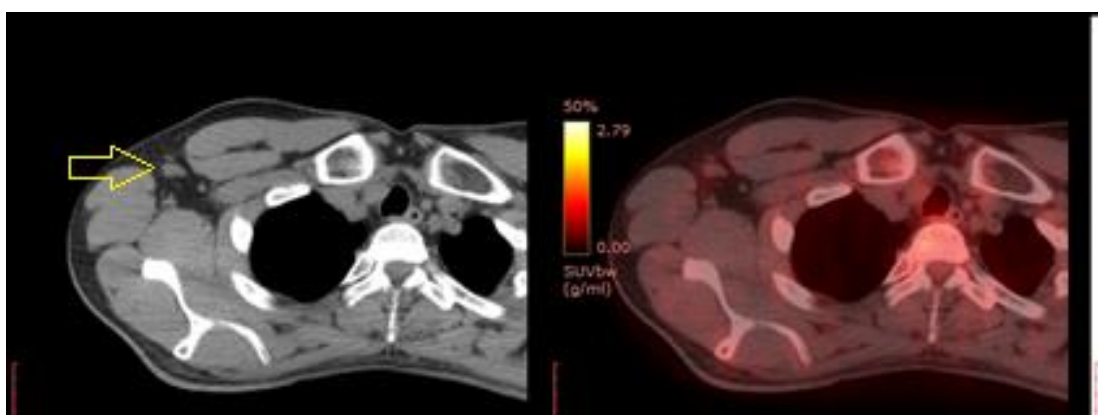

Fig. (2)(b): Enlarged right axillary lymph node measuring about $18 \mathrm{~mm}$ with no tracer uptake. 

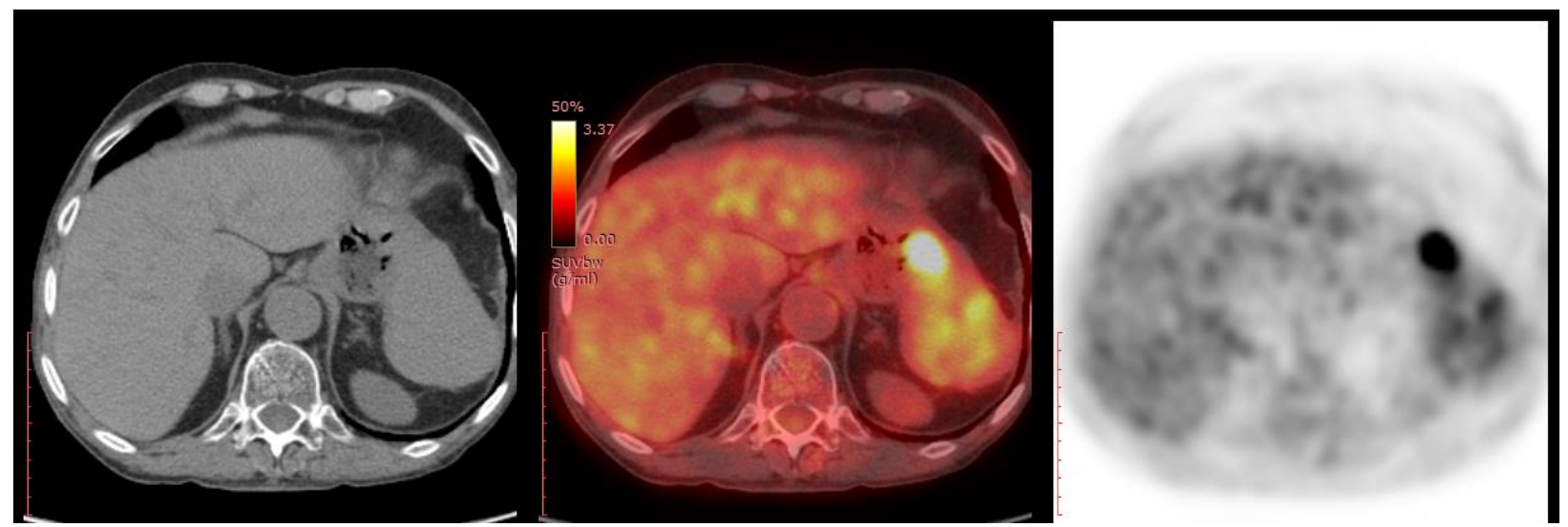

Fig. (3)(a): Average gastric wall thickening with active focal nodular lymphomatous infiltrations at fundal region and an inseparable from upper middle splenic pole measuring about $(2.5 \times 2 \mathrm{~cm})$ with $\mathbf{S U V m a x}$ of 8.
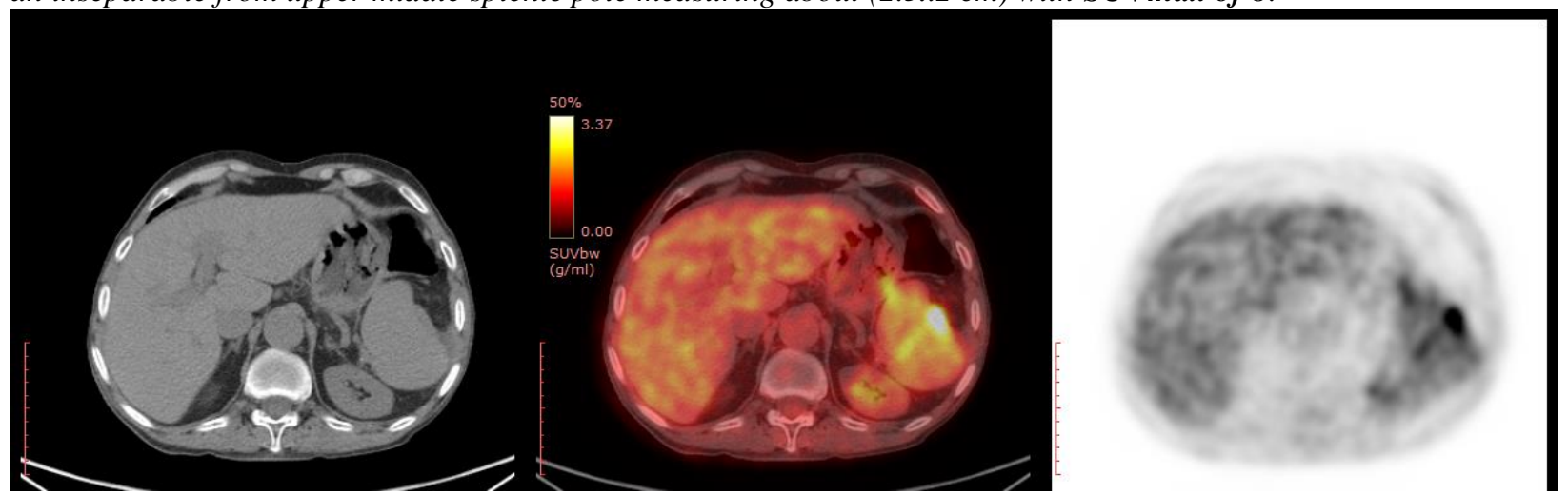

Fig. (3)(b): Focal lymphomatous infiltrates noted related to the splenic hilum measuring about $(2 \mathrm{~cm})$ with $\mathbf{S U V m a x}$ of 5.

\section{Discussion}

PET/CT has an advantage over CT/MRI because of its ability to distinguish between viable tumor and necrosis/fibrosis in residual masses after treatment, provided the pretreatment scan showed the tumor to be FDG avid (5).

In current study, we had 80 patients referred for initial assessment of Non-Hodgkin lymphoma, their SUVmax ranged from $3.0-$ 49.0 with mean value of $17.05 \pm 11.86 \mathrm{SD}$, the SUVmax in aggressive type of NHL ranged from $3.0-49.0$ with mean value of $22.01 \pm 9.88 \mathrm{SD}$ and that of indolent NHL ranged from 3.3 - 14.0 with a mean value of $7.84 \pm 3.05$, where aggressive type of NHL is significantly higher $(\mathrm{p} \leq 0.01)$ than in indolent type.

A previus study by (6) showed that an FDG uptake of more than $10 \mathrm{SUV}$ is predictive of an aggressive B-cell lineage or suggestive of the presence of a more aggressive histological component It may thus play an important role 
in identifying the foci of aggressive transformation or aggressive histology in those patients who were thought to harbor an indolent lymphoma on previous biopsy. Thus having an important diagnostic and therapeutic implications .Another study (7), also state that PETCT has the potential to detect transformation of a low-grade nonhodgkin lymphoma to a more aggressive subtype (Richter transformation) based on the degree of FDG avidity.

In general, many studies have shown that low grade or indolent lymphomas are less FDG avid than are high-grade or aggressive types. In NHL, diffuse large B-cell lymphoma and grade 2 or 3 follicular lymphoma demonstrate the highest FDG metabolism. This usually translates into a threefold higher standardized uptake value (SUV) than in a low- grade follicular, marginal zone, or small cell lymphoma, in which the SUVs tend to be lower (8).

Among the 40 patients referred for follow up assessment in the present study, we found that their baseline SUVmax PET/CT examination ranged from 3-49.0 with a mean value of $17.05 \pm 11.86 \mathrm{SD}$, with a significant change $(p \leq 0.046)$ in their follow up examination with SUVmax ranged from $0.0-$ 28.0 with a mean value of $11.73 \pm 9.25 \mathrm{SD}$.
According to the International Harmonization Project Criteria for Assessment of Response to Therapy of Lymphoma, the change of the FDG uptake in a lesion (which was FDGAvid at Baseline) was included in the criteria to determine the response to treatment assessment, where complete remission is assigned to complete resolution of FDG-avid lesions regardless to the presence of a residual mass on CT while partial remission is determined by $>50 \%$ decrease in sum or product of diameters of lesions with persistent residual FDG uptake in at least one site, whereas progressive disease is attributed to any new FDG-avid lesion and finally stable disease is where there is no complete remission, partial remission, or progressive disease (9).

In our study, among the 80 cases referred for initial staging and according to the Ann Arbor classification of Non-Hodgkin's Lymphoma, the cases were staged as follows; CECT detected $8(10 \%)$ patients in stage I, 2 patients in stage IE (2.5\%), 24 (30\%) patients in stage II, 6 patients (7.5\%) in stage IIE, $20(25 \%)$ patients in stage III and 20 (25\%) patients in stage IV. The PET/CT detected 6 (7.5\%) patients in stage I, 16 (20\%) patients in stage II, 8 patients (10\%) in stage IIE, 12 patients (15\%) in stage III, 4 patients (5\%) in stage IIIE, $34(42.5 \%)$ patients in stage IV and 
significantly identified $(\mathrm{p} \leq 0.001)$. Thus, PET/CT agreed with CECT in 38 cases (47.5 $\%)$ and disagreed with it in $42(52.5 \%)$ cases, where PET/CT upstaged 34 cases and down staged 8 cases.

In a previous study (10), PET/CT was used in initial staging of 41 cases. PET/CT and conventional imaging methods were concordant in the staging of 30 patients $(73.2 \%)$ and discordant in remaining 11 patients $(26.8 \%)$. PET/CT upstaged 5 patients (12\%) out of the 11 discordant cases and down-staged 6 patients $(14.5 \%) .2$ patients were upstaged from stage I to stage II. 1 patient from stage II to stage III. 2 cases $(4.8 \%)$ were upstaged to stage IV, 1 patient from stage II to stage IV.

In another study (11), noted that PET/CT mostly upstaged disease when compared with CECT alone and this was observed mostly for stages I and II for. In the more advanced stages III and IV, PET/CT may not have any added value if the disease was already defined as advanced by a diagnostic CT alone. From the 138 patients in this study, 18F-FDG avid subcentimetric lymph nodes were detected in 16 patients, these additional sites of lymphomatous involvement would have been missed if staging was done using CT alone. In 10 patients there were no changes in the staging as they were already stage III and IV, while in 6 patients $(4.5 \%)$ the detection of the 18F- FDG avid subcentimetric lymph nodes resulted in restaging of these patients from stage I into II and stage II into III.

PET/CT impacts not only the staging, but also the therapy protocol, in lymphoma staging, PET/CT tends to upstage rather than downstage tumors (11).

Staging of lymphomas is a vital pre-requisite to their appropriate therapeutic management and prognostication. This is based on the Ann Arbor system, to which has been added a definition of bulky disease called the Cotswold modification. Upstaging by PET/CT for NHL included the detection of increased FDG uptake in normal- sized lymph nodes (usually less than $1 \mathrm{~cm}$ ) as well as in extra-nodal sites, previously missed by diagnostic CT but nevertheless FDG avid. In a few cases, paraspinal and pulmonary lesions interpreted as benign by the CT scan were read as involved according to the PET/CT (11). Down staging patients because of computed tomography-positive but PET/CT negative lesions should be performed carefully. 18 F-FDG PET/CT does not replace conventional staging techniques but adds useful information (9). 
Several studies compared CECT to $18 \mathrm{~F}-\mathrm{FDG}$ $\mathrm{PET} / \mathrm{CT}$ in detection of extra-nodal lymphoma; all were around $88 \%$ sensitivity and $100 \%$ specificity for 18F-FDG PET/CT compared to $50 \%$ sensitivity and $90 \%$ specificity with CECT (3).

In our study, among the 40 patients referred for follow up assessment after treatment according to the size in CECT and FDG activity in PET/CT ; CECT showed 14 patients $(35 \%)$ in complete remession, 18 patients $(45 \%)$ in regressive disease, 4 patients $(10 \%)$ in stable disease and 4 patients (10\%) in progressive disease while PET/CT showed 16 patients $(40 \%)$ in complete remission, 20 patients $(50 \%)$ in partial regression, $2(5 \%)$ patients in stationary disease , $2(5 \%)$ patients in progressive disease.

In a large retrospective study by (12), they studied 151 patients with mediastinal lymphoma, 57 cases of HD (37.8\%) and 94 cases of NHL (62.2\%). The follow-up program for each patient included 18F-FDG PET every 6 months for the first 2 years and then every 12 months for a further 3 years. Positive 18F-FDG PET results suggested lymphoma relapse in 30 of 151 patients after the completion of therapy (19.8\%). Histology confirmed relapse in 17 of the 30 patients (true positive in $56.7 \%$ ), whereas either a benign condition (fibrosis) or sarcoid-like granulomatosis or an unrelated neoplasm (a thymoma) was demonstrated in the remaining 13 patients. Only 3 of the 17 patients with relapse had symptoms or signs suggesting relapse at the time of the positive.

18F-FDG PET scan. 27 patients (90\%) had concurrent CT and PET positive results, while only 3 patients (10\%) proved PET/CTpositive and CT-negative, in all these 3 patients, the histopathological findings were positive for lymphoma relapse.

Many studies have shown that 18F-FDG PET performed after treatment is highly predictive of progression free survival and overall survival in HL and aggressive NHL with or without residual masses on $\mathrm{CT}$. It seems that functional imaging with 18F-FDG PET enables evaluation of the earlier metabolic changes rather than the anatomic changes of the lymphoma and distinguish between viable lymphoma cells and necrosis or fibrosis in residual masses after treatment. On the basis of these findings, the International Harmonization Project criteria for reviewing PET scans were based on visual interpretation and intended for end-of-treatment evaluation (9). 


\section{Conclusion}

PET/CT proved statistically to be more efficient in follow up studies after chemotherapy using the standard uptake value (SUV) being more related to the functional activity of the residual tumor cells rather than to the size of the tumor itself.

\section{References}

1. Ninkovic S, Lambert J. Non-hodgkin lymphoma. Medicine (Baltimore). 2017;45(5):297-304.

2. Terezakis SA, Yahalom J. PET-computed tomography for radiation treatment planning of lymphoma and hematologic malignancies. PET Clin. 2011;6(2):165-75.

3. Terasawa T, Nihashi T, Hotta T, Nagai H. 18FFDG PET for posttherapy assessment of Hodgkin's disease and aggressive Non-Hodgkin's lymphoma: a systematic review. J Nucl Med. 2008;49(1):13-21.

4. Kwee TC, Kwee RM, Nievelstein RAJ. Imaging in staging of malignant lymphoma: a systematic review. Blood, J Am Soc Hematol. 2008;111(2):504-16.

5. Hochhegger B, Alves GRT, Irion KL, Fritscher CC, Fritscher LG, Concatto NH, et al. PET/CT imaging in lung cancer: indications and findings. J Bras Pneumol. 2015;41:264-74.
6. Ngeow JYY, Quek RHH, Ng DCE, Hee SW, Tao M, Lim LC, et al. High SUV uptake on FDGPET/CT predicts for an aggressive B-cell lymphoma in a prospective study of primary FDG-PET/CT staging in lymphoma. Ann Oncol. 2009;20(9):1543-7.

7. Schöder H, Moskowitz C. PET imaging for response assessment in lymphoma: potential and limitations. Radiol Clin North Am. 2008;46(2):225-41.

8. Elstrom R, Guan L, Baker G, Nakhoda K, Vergilio $\mathrm{J}-\mathrm{A}$, Zhuang $\mathrm{H}$, et al. Utility of FDG-PET scanning in lymphoma by WHO classification. Blood, J Am Soc Hematol. 2003;101(10):3875-6.

9. Juweid ME, Stroobants S, Hoekstra OS, Mottaghy FM, Dietlein M, Guermazi A, et al. Use of positron emission tomography for response assessment of lymphoma: consensus of the Imaging Subcommittee of International Harmonization Project in Lymphoma. J Clin Oncol. 2007;25(5):571-8.

10. Riad R, Omar W, Kotb M, Hafez M, Sidhom I, Zamzam M, et al. Role of PET/CT in malignant pediatric lymphoma. Eur J Nucl Med Mol Imaging. 2010;37(2):319-29.

11. Raanani P, Shasha Y, Perry C, Metser U, Naparstek E, Apter S, et al. Is CT scan still necessary for staging in Hodgkin and nonHodgkin lymphoma patients in the PET/CT era? Ann Oncol. 2006;17(1):117-22.

12. Zinzani PL, Tani M, Trisolini R, Fanti S, Stefoni V, Alifano M, et al. Histological verification of positive positron emission tomography findings in the follow-up of patients with mediastinal lymphoma. Haematologica. 2007;92(6):771-7.

To cite this article: Hesham E. ELsheikh, Mohamed A. Attia, Adel M. Kotb, Role of Positron Emission Tomography/Computed Tomography in The Initial Staging And Evaluation Of The Therapeutic Response in Patients with Non-Hodgkin Lymphoma. BMFJ 2022;39 (Radiology):1-13. DOI: $10.21608 / \mathrm{bmfj} .2021 .83781 .1438$ 